\title{
Positioning Improving of RSU Devices Used in V2I Communication in Intelligent Transportation System
}

\author{
Marzena Banach ${ }^{1,2}$ Katarzyna Kubiak $^{1,3}$ and Rafał Długosz ${ }^{1,4}$ \\ 1 Aptiv Services Poland \\ ul. Podgórki Tynieckie 2, 30-399, Kraków, Poland \\ 2 Poznan University of Technology, Institute of Architecture and Spatial Planning, \\ Nieszawska 13C, 61-021 Poznan, Poland \\ E-mail: marzena.banach@erba.com \\ ${ }^{3}$ Adam Mickiewicz University \\ Faculty of Mathematics and Computer Science \\ ul. Uniwersytetu Poznańskiego 4, 61-614 Poznan, Poland \\ E-mail: kbkubiak45@gmail.com \\ ${ }^{4}$ UTP University of Science and Technology \\ Faculty of Telecommunication, Computer Science and Electrical Engineering \\ ul. Kaliskiego 7, 85-796, Bydgoszcz, Poland \\ E-mail: rafal.dlugosz@aptiv.com, rafal.dlugosz@gmail.com
}

\begin{abstract}
In this work we present solutions which aim at enhancement of the localization precision of the road side unit (RSU) devices which will participate in vehicle-to-infrastructure (V2I) communication in future autonomous driving and intelligent transportation systems (ITS). Currently used localization techniques suffer from limited accuracy which is due to various factors, including noise, delays caused by environmental conditions (e.g. temperature variation) and differences in elevation between devices communicating with each other in the road environment. In case of application of the ITS, these factors can be the source of significant discrepancies between real positions of the RSUs and their estimated values provided by the V2I system. The proposed techniques, based on various approximation techniques, as well as linear and nonlinear filters, allow to improve the localization accuracy, reducing the positioning errors by more than $90 \%$.
\end{abstract}

Keywords - Intelligent Transportation System, Smart City, V2I communication, Automotive Active Safety

\section{INTRODUCTION}

An intelligent transportation system (ITS) is a relatively new concept which embraces solutions whose aim is to optimize transport based on modern technologies. They include those in the field of artificial intelligence (AI) as well as information and communication technologies (ICT).

Functionalities considered under the framework of the ITS can be classified into several general classes. One of them are solutions responsible for providing appropriate, up-to date information for passengers of public transport and car drivers. Another group aims at increasing traffic flow in urban areas in order to eliminate or reduce the traffic jams, which can cause a significant reduction in pollution levels in the cities. Finally, one of the main ITS development directions are the vehicles themselves. It is supposed and hoped that the majority of the vehicles in the nearest future will be equipped with advanced driver assistant systems (ADAS). However, future development of the ITS may be understood as the development of autonomous vehicles which will enable traveling without human intervention. The purpose of the development of such vehicles is to improve road safety and ecological aspects.

Taking into consideration the last group of solutions, several essential challenges can be indicated here. As the car moves through the city or suburban/highway areas, the surroundings of the vehicle are dynamically changing. One of the main problems is high complexity of the environment seen by the vehicle. This makes the algorithms responsible for travel safety also complex. The complexity means, for example, a number of objects in the range of the vehicle's sensors, both moving and still objects, their trajectories, obscuring some objects by others, etc. One of the possible solutions in terms of the described problem can be intelligent road infrastructure providing direct support for the vehicle movement, in the form of special devices mounted at fixed points of the road and urban environment.

The support can be provided by the so-called vehicle-tovehicle $(\mathrm{V} 2 \mathrm{~V})$ and vehicle-to-infrastructure (V2I) communication (V2X in short). For example, the V2I system facilitates the operation of traffic sign recognition (TSR) functions. In case of low visibility of traffic signs (TSs), the information passed to the vehicle wirelessly by devices (RSU - road side unit) associated with particular TSs, or groups of them, can significantly improve the performance of such functions. V2I techniques can be used to inform the driver about accidents ahead, bad road conditions, etc. In its most advanced form, a framework of the RSUs participates in building the so-called model (map) of the environment of the vehicle. In the currently 
used ADAS systems, the map of the environment is created by the vehicles themselves, which use their own on-board sensors (camera, radar, recently LiDAR) and appropriate algorithms. The V2I system can be treated here as an additional sensor which provides additional data, for example from "behind the corner",

One of the main challenges in the described problem is the need of very accurate localization of the RSUs by the passing vehicle. This can be based on a real time localization system (RTLS). Such systems are frequently used in indoor confined areas (buildings) for various purposes. They are usually composed of two types of devices: moving devices (active markers/tags) as well as devices mounted in fixed points of buildings (transponders/anchors). Trajectories of the markers are determined and recorded by the anchors on the basis of multiple distance measurements between particular markers and the framework of the anchors, supported by the trilateration computation techniques [1].

In the indoor systems, the positions of the anchors are usually well known. In case of the urban/road environment, on the other hand, the situation is different. The RSUs can be viewed as counterparts of the anchors, while the markers are the devices associated with the moving vehicles. Contrary to typical indoor applications, it is the device mounted in the vehicle which determines own position in the ITS. Another difference is the lack of fixed framework of the anchors, as the moving vehicle on its path is within the range of different RSUs. Due to these differences, the computation techniques used to determine the relative positions of the vehicles have to be different as well.

We assume that the moving vehicle is able to calculate its own trajectory, within given accuracy, on the basis of its own GPS unit and own on-board sensors (yaw rate and velocity sensors). Due to the noise as well as low precision of the applied sensors, the computed trajectory is also not precise. Constant communication with the RSU devices, which as we assume, know their own positions in the global coordinate system (GCS) increases the precision of the calculated trajectory of the car. The main issue here is how to precisely determine the trajectory in relation to the framework of the RSUs. It is the topic of the presented work.

\section{STATE-OF-THE ART STUDY}

In this part we briefly present state-of-the art study in the areas related to the presented topic. As an example of a system which can benefit from the proposed method, we consider the traffic sign recognition (TSR) system which is becoming a standard in the automotive industry, including European New Car Assessment Program (Euro NCAP) [2]. Currently used systems of this type rely only on the on-board sensors (cameras) of a vehicle. Traffic signs (TS) are then identified and recognized using various signal processing and artificial intelligence (AI) methods. The computation scheme is in general similar in each system of this type. Firstly, the TSs are identified in the images taken from the camera, then they are cropped to smaller images and normalized in terms of sizes. On the basis of a series of frames obtained from a single camera or on the basis of stereo-vision cameras, the positions of the TSs in the environment of the vehicle are determined using, among others, the trilateration methods. The cropped and normalized TSs are then provided to an AI algorithm for identification.

\section{A. Towards automotive TSR systems of the $2^{\text {nd }}$ generation}

The TSR systems currently offered on the market recognize only selected traffic signs, usually those related to speed limitations, stop signs, etc. In the future, with the development of fully autonomous cars, one can expect that these systems will be able to recognize almost all TSs, similarly to the human driver. This will strongly increase the complexity of the implemented algorithms. The problem which can be frequently observed on the roads is the non-standard appearance of the road signs (damage, coverage, lack of full exposure, suspension at non-standard height, etc.), as shown in Fig. 1. Additionally, taking frequently observed dense arrangement of the TSs over a given area into account, it can lead to various safety issues if the vehicle will only rely on its on-board sensors.

Various efforts which aim at solving the problem of the road sign visibility can be found in the literature. One of the proposed solutions is inclusion of the TSs in the future system supported by the V2I communication. In practice, it means equipping the TSs with the RSU devices capable of transmitting relevant information to passing vehicles. A proposal of such a solution is described, for example, in [3], [4] and [5]. These solutions can be regarded as a next important stage toward the development of active traffic signs.

One of the problems here is how to properly determine the positions of the RSUs associated with their respective TSs in the situation when many TSs are located in close proximity to each other. An exemplary situation of this type is shown in Fig. 2. If the positioning/localization of a TS is not perfect, it can lead to false assignments of the RSUs to the TSs seen by the vehicle and thus to false behavior of the active safety (AS) system of the vehicle.

Precise positioning of the RSU devices will be of great significance for applying them as support in creating a dynamic map of the environment of the moving vehicle.

\section{B. Applications of the RTLSs - desired parameters}

The RTLSs are being developed and the localization accuracy is being improved around the world. The majority of these investigations focus on indoor applications [6], [7].

The desired ranging precision (requirements) always depends on the application for which a given system is designed. In some medical applications, even millimeter precision is mandatory. Such a situation takes place in motion capture systems which aim at recording the motion pattern of a disabled person with very high accuracy [8], [9]. Another example is warehouse application for which the precision of $20-50 \mathrm{~cm}$ is acceptable. An RTLS of this type is offered by the Ubisense Company [10], with the reported localization errors at the level of $15 \mathrm{~cm}$. This system was designed to operate on relatively large areas. The reported localization errors in 

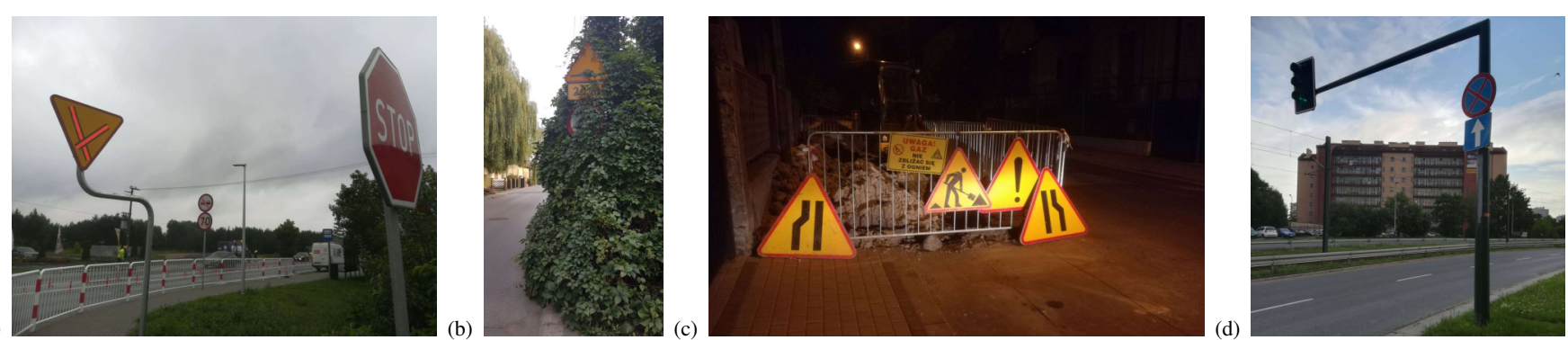

Fig. 1. Examplary real road situations illustrating: (a, b) road signs deviating in appearance from standards or invisible, (c, d) untypical elevation at which a TS can be mounted (own source).
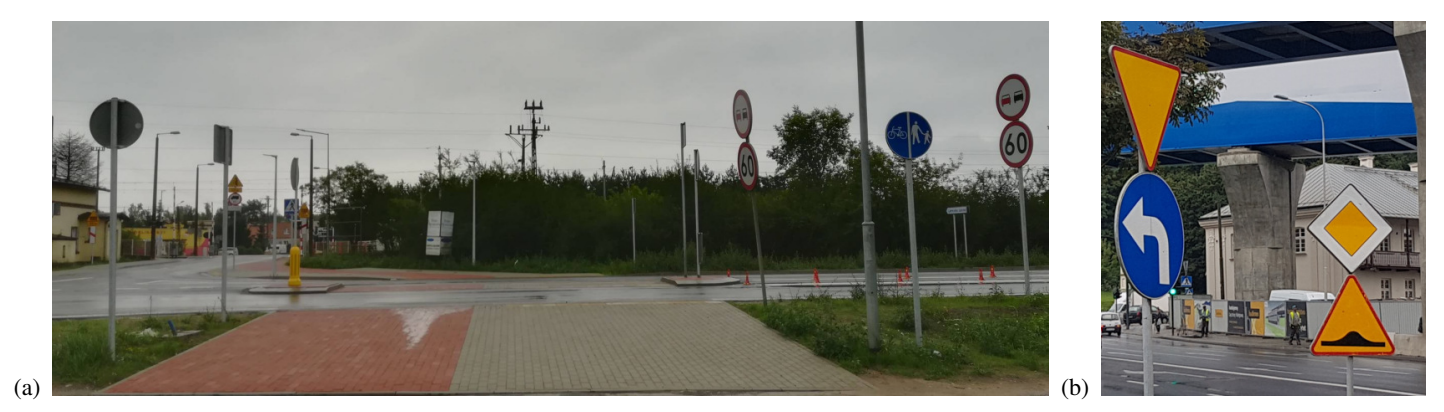

Fig. 2. E

xemplary real road situations illustrating large density of traffic signs in a given area (own source).

indoor conditions are usually below $10 \mathrm{~cm}$ [11]. This however, is achievable for relatively small distances of below $10 \mathrm{~m}$. The required precision of the ITS will be different, depending on the target application of the ADAS.

The localization precision is not the only parameter to be considered and optimized. In the above-mentioned indoor human motion capture system the most important problems include improving the ranging precision toward even submillimeter range, and miniaturizing the markers to make them comfortable for the examined persons. As these devices are wearable, the energy consumption is also a crucial factor. On the other hand, such systems will be used in the indoor environment, in which temperature is at an almost equal level. Therefore, the robustness to a wide-range temperature variation is rather less important. Normally, in systems of this type, the RTLS tags are affixed to moving objects and tracked by the transponders installed in the fixed points of the environment, with precisely determined (and stored in the memory) positions. The net of transponders may thus create a precise frame of reference for the moving markers. The transponders have access to a power supply (or can be battery operated) and therefore a strong miniaturization is not a critical factor. Additionally, the framework of anchors can take a sufficiently long time to calibrate precisely.

When the RTLS is applied in the ITS, the situation is substantially different from the typical indoor conditions. In the road environment, the network of devices communicating with each other covers a much wider area than in the described indoor systems. Also, the behavior of such systems in cities can differ from the one in suburban areas, mostly due to different device densities in these areas. In the suburban areas, a rather sparse network of the RSU devices is expected, with a small number of devices in the range of the vehicle sensors. This creates some problems in terms of the localization precision, but in some situations it can also simplify the communication scheme (less interference).

\section{RTLS-based solutions in Intelligent Transportation Systems}

The use of the impulse-radio ultra wideband (IR-UWB) localization technology in an outdoor environment has already been proposed [12], [13], [14], [15]. This technology provides high channel capacity (i.e. the data is transmitted at a high rate) which makes is suitable for use in the V2I communication [12]. Also, propositions to employ the IR-UWB technology in the positioning of the objects on the road have been reported [13].

In sparse and open networks which are expected in the suburban areas, there will be only two devices communicating with each other in the worst case scenario. One of them will be an on-board unit (OBU) of a moving vehicle. The second one - the RSU - will be mounted in a fixed position of the road infrastructure. The trajectory of the moving vehicle can be determined, with a relatively good accuracy, on the basis of the vehicle velocity, acceleration, the yaw rate and other kinematic parameters. A single communication session between the OBU and the RSU will be quick enough to assume that both devices are in still positions during this period, which can be determined using only basis kinematic equations. Let us consider an example: if the distance between both communicating devices is even at the level of $100 \mathrm{~m}$, the velocity is even $100 \mathrm{~km} / \mathrm{h}(22.5 \mathrm{~m} / \mathrm{s})$, then the distance traveled 
during a single communication session does not exceed 20$25 \mu \mathrm{m}$. This value is far below the safety margin and thus can be neglected.

\section{ENHANCEMENT OF THE RSU POSITIONING - PROPOSED SOLUTIONS}

In the investigated method, the vehicle measures its distance to the RSU frequently (a time-of-flight (ToF) approach). On the basis of a single measurement session only the distance, $r$, can be determined, while the azimuth and thus the position of the RSU are not known. As a result, a circle with the radius $r$ is obtained. The RSU is situated somewhere on it. The vehicle is located in the central point of the circle. Theoretically, on the basis of only two measurement sessions (for different car positions) and the trilateration computation, it is possible to determine the position of the RSU in relation to the vehicle.

In practice, various negative factors can interfere with the measurements. Among them are: delays caused by the communicating devices, noise resulting from imperfections of the vehicle on-board sensors, unknown height over the road surface, at which the RSU device is mounted, etc. All these factors result in the measurements being subject to errors. Depending on the source of these errors, they can be either systematic or random.

The problem is illustrated by an exemplary simplified trajectory of the vehicle in Fig. 3. The left diagram shows selected positions of the vehicle, for which distance measurements are performed. The results for different delays introduced by both of the communicating devices are shown. Large black circles illustrate an ideal case, in which delays are known (nominal values). In this case, all obtained circles (marked as "real distances") after a correction by a known factor intersect at a single point, which is the real position $\left(x_{\mathrm{R}}, y_{\mathrm{R}}\right)$ of the RSU device.

Theoretically, basing on the trilateration method, the position of the RSU device can be determined from the results of only two measurements, as mentioned above. However, under real conditions, unknown signal delays cause that the resultant circles (marked as "measured distances") intersect in other points (seeming positions). The right diagram in Fig. 3 shows the results for delays deviating from the nominal value towards both positive and negative values. Both types of deviations are shown here for illustration only. In the real situation, only one type will occur. Therefore, in the following figures only single circles are shown for each distance measurement.

The obtained points of intersection $(\mathrm{A}, \mathrm{B}, \ldots, \mathrm{J})$ for given values of delay form the area of uncertainty, with the unknown position of the RSU within it, as shown in the right diagram of Fig. 3. The uncertainty at the level of $10 \mathrm{~ns}$ can cause the localization error of even $\pm 1.5 \mathrm{~m}$, assuming the speed of light $v_{\mathrm{c}} \approx 30 \mathrm{~cm} / \mathrm{ns}$, and two-way communication. Assuming that an error of this magnitude is the case in a suburban area and that the considered system recognizes TSs, then the error could be neglected as the distance between any two TSs is assumed to be significantly greater than the error. In contrast, such error would be too big to be neglected in urban areas or when the RTLS is used to build a dynamic model of the environment.

\section{A. Steps of the proposed enhancement algorithm}

The proposed method for estimating the RSU position consists of several stages. In practice, it is an iterative method, where particular steps are performed cyclically and alternately for every new measurement session. The obtained data sets can be kept in memory as a whole or be realized as a delay line (a shift register), as for example in finite impulse response (FIR) filters.

The method described below was implemented in the Octave environment and verified for different values of particular factors and different trajectories of the vehicle. In order to model real conditions in a better way, which is expected during the driving, the measurement values were artificially disturbed by noise with different amplitudes, $A$, as presented below.

Stage 1: Distance measurements $\rightarrow$ data set with seeming positions of the RSU

For every new, $i^{\text {th }}$, measurement and the new resultant circle with the radius $r_{i}$, new intersection points are computed using the circle determined in the previous iteration.

The objective of this stage is to compute the seeming position of the RSU device (i.e. spatial $x$ and $y$ coordinates). Let us denote the seeming position of the RSU as SPR. $x, y$. The determined SPRs can be expressed in the global coordinate system (GCS), which simplifies the computations. However, any coordinate system used consistently can be applied as well. The vehicle position can also be expressed in GCS.

It is worth to notice that this method returns a pair of intersection points, so it is necessary to specify which of them is the one reflecting the real position of the RSU. Basing on only two measurements, it is not possible. However, for a larger number of measurements, and thus a larger data set of calculated SRSs, the RSU position can be deduced. If the trajectory of the vehicle is not a perfectly straight line, then the points which are the real equivalents of the RSU position are spatially more focused and form an area which reflects a regular circle (or arc), as shown in Fig. 3. On the other hand, if the vehicle's trajectory would be a straight line, then even larger number of measurements do not resolve the ambiguity problem. Such a situation may happen in urban areas, where straight line movements of the vehicle will be common. Certain amount of curvature in the vehicle's movement is therefore needed. The described ambiguity problem may be solved in other ways, for example through content of messages provided by the V2I system to the vehicle. The vehicles may also intentionally focus only on TSs on one of the road sides.

Stage 2: Seeming positions of the RSU $\rightarrow$ data set with estimated positions of the RSU

The seeming positions of the RSU (SPRs) form an area resembling a circle, with the radius $r_{\mathrm{SC}}$. What makes the approximation task more difficult is the fact that only a portion of the circle is obtained. How well the points match the circle/arc depends on several factors. A good-quality image of the circle is obtained in the absence of noise and with sufficiently dense measurements. As already described, the 

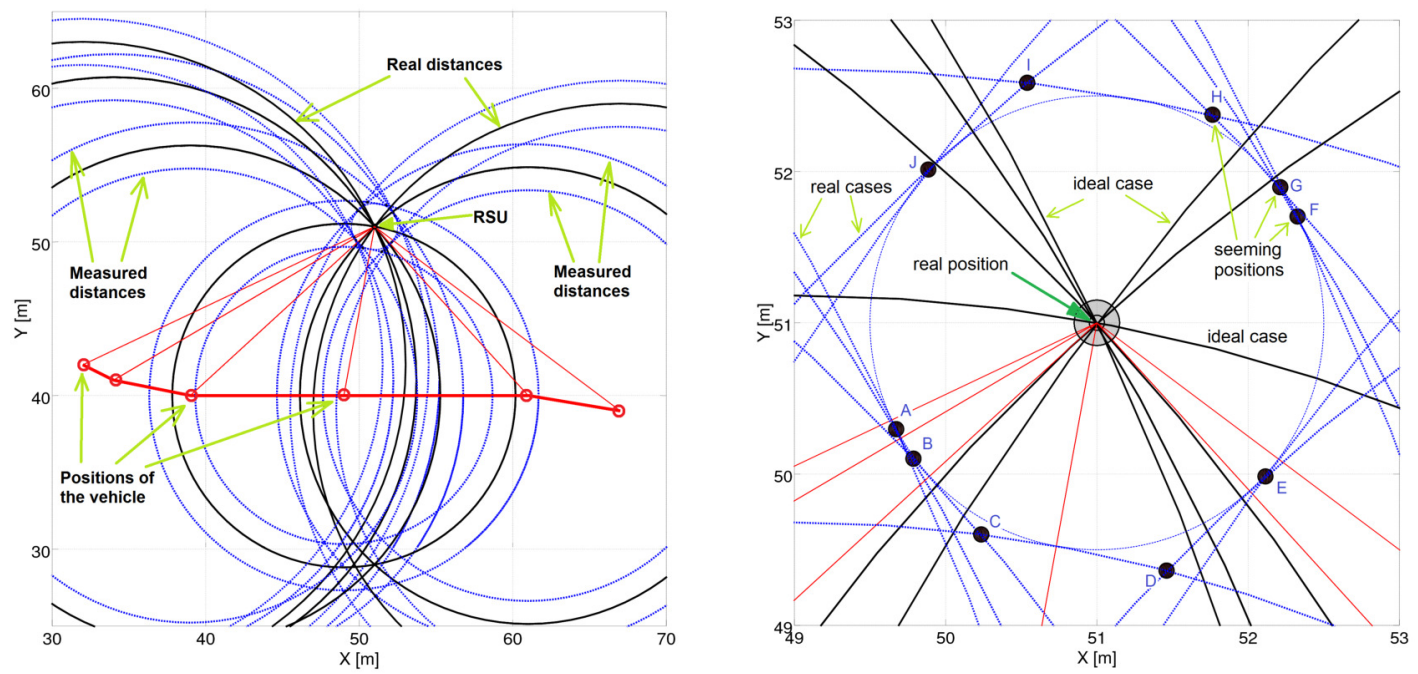

Fig. 3. Determining the RSU position on the basis of multiple measurements - a general idea.

(a)
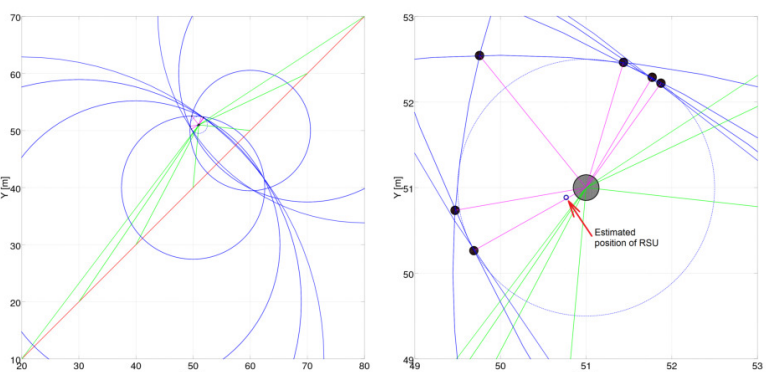

(b)

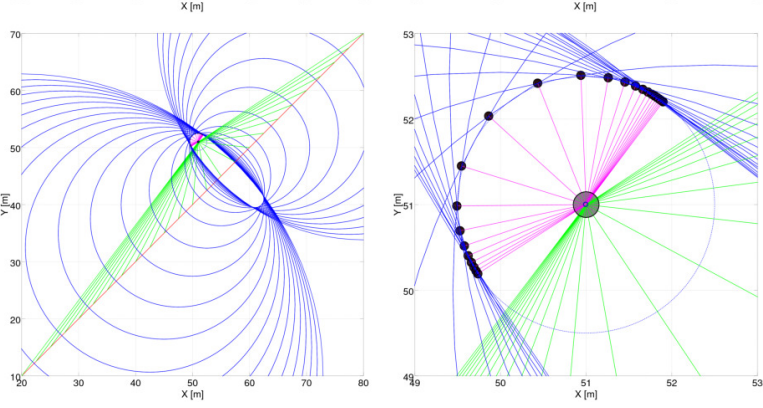

Fig. 4. Selected results for particular measurement frequencies.

objective is to obtain a high density of the SPRs relative to the azimuth angle. If the RSU is far ahead of the vehicle and the vehicle is apporaching it, then the azimuth angle $\alpha$ does not change substantially, and thus the measurements can be less frequent in time. On the other hand, if the vehicle is in a closer proximity to the RSU mounted at the side of the road, then the azimuth varies faster for a given velocity of the vehicle. The investigation results show that $\Delta \alpha$ at the level of $10^{\circ}$ is sufficient.

The impact of the selected frequency of the measurement on the accuracy of the computed positions of the SPRs is illustrated in Fig. 4 for constant velocity of the vehicle. In this case the measurements are performed at equal time intervals. In Fig. 4 (a) the measurement density is too small, causing the image of the circle to be distorted. This leads to larger final errors, as marked with the arrow. For a better illustration we set the noise amplitude to zero in this case.

In real situations the noise is non-zero and the SPRs are spread across the edge of the circle. In this situation the parameters of the circle (its radius, as well as the $x$ and $y$ coordinates) have to be determined. This is performed in the second stage of the proposed method. The tests were performed with the noise of different amplitudes. Noise samples were generated according to the uniform distribution with the extreme values between $-A$ and $A$.

Stage 1 provides the data set with calculated SPRs. Stage 2 introduces an approach which relies on estimating the circle parameters basing on three selected points, which are assumed to be located on this circle. In case of a high angular density of the distance measurements, particular SPRs can be located very close to each other. To minimize the impact of the noise on the estimation results, the points are selected so that they are not too close to each other. For the purposes of the presented method, existing methods of this type were adapted and some modifications were introduced.

Stage 3: Filtering over the data set of the estimated positions of the RSU

Stage 2 provides a new dataset with computed intermediate seeming circles, representing estimates $\left(x_{\mathrm{E}}, y_{\mathrm{E}}\right)$ of the real position of the RSU $\left(x_{R}, y_{R}\right)$. The size of this set can increase with each new distance measurement or be constant as in the delay line used in filters. Depending on the amplitude of noise, the obtained intermediate circles can significantly differ from each other, in terms of both the position and the radius. The objective of this stage is to find an appropriately averaged circle, with a supposed real position of the RSU.

In this (final) stage various operations can be performed including: sorting, filtering, truncating, etc. Multiple tests $(10,000+)$ allowed us to determine a method and its settings 
(a)

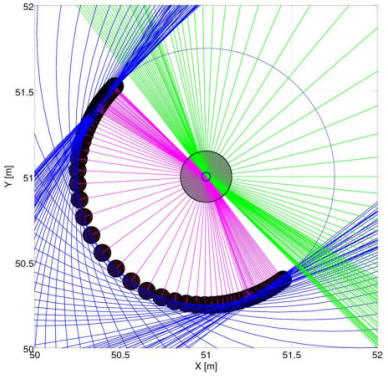

(c)

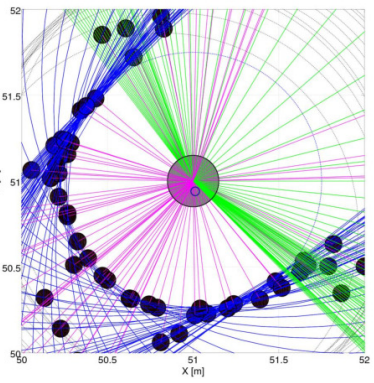

(d)

(b)
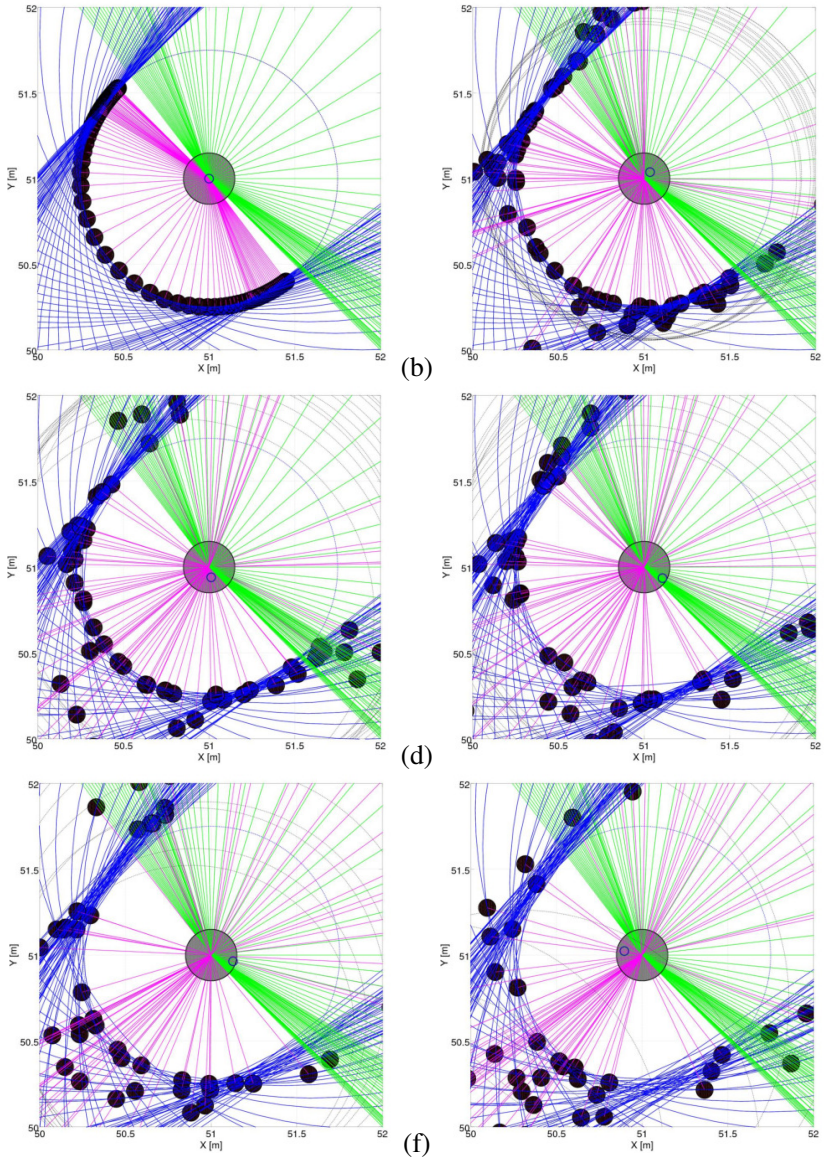

Fig. 5. Estimated positions of the RSU for time delay of $5 \mathrm{~ns}(1.5 \mathrm{~m})$ and different values of the noise (amplitude $A$ ) applied to the measured distance, for : (a) $A=0[\mathrm{~mm}]$, (b) $A=150[\mathrm{~mm}]$, (c) $A=300[\mathrm{~mm}]$, (d) $A=$ $450[\mathrm{~mm}]$, (e) $A=600[\mathrm{~mm}]$, (f) $A=750[\mathrm{~mm}]$.

which provide a relatively good approximation of the positions of the RSU devices, including the case of noise with relatively large amplitude.

The dataset which results from Stage 2 can contain biased data (i.e. circles with very large radius values). Therefore, we initially remove all circles whose radii are greater than fixed radius threshold value (for example, 3 meters) in order to ensure the reliability of the data. Also, the extreme case when all circles are larger than the fixed threshold should be not be analyzed. The threshold value can be adjusted on the basis of statistical analysis of the obtained results.

After the initial data verification we sort the remaining dataset separately over the $x$ and the $y$ coordinates of the middle points of the circles. We observe that usually a large portion of the dataset with the circles is located in the proximity of the real position of the RSU. After the sorting operation, these circles are located in the middle of the dataset, for both the $x$ and $y$ coordinates, while those that have totally false values (in terms of $x$ and $y$ coordinates) are located on both peripheral areas of the set. In one of the tested scenarios, a smoothing low pass FIR filtering over the sorted dataset was applied. In these tests, filters with equal coefficients of the order $N=1,2$ or 3 were used. This operation slightly

improved the results. However, to come to a more general conclusion and to avoid false positives, further investigations are required.

The sorted and optionally filtered datasets are truncated at both sides, taking into account some additional parameters. One of them is the spread (threshold) across the median value of the remaining dataset. The threshold can be set to be a constant parameter or be determined, for example, on the basis of the variance across the median value.

Finally, the $x_{\mathrm{E}}$ and $y_{\mathrm{E}}(\mathrm{E}$ - estimated) coordinates of the RSU are found as the median values of the output dataset. The calculated position is still only an approximate of the real position. However, the obtained results, shown in Fig. 5, are substantially better than those expressed only by the SPRs, computed in Stage 1. Comprehensive investigations performed for different values of particular parameters (trajectories of the vehicle, noise levels, delay times, etc.) show that the determined positions can be improved by more than $90 \%$. This value was determined by comparing two quantities for each performed test. One of them is the radius of the seeming circle, $r_{\mathrm{SC}}$, resulting from the assumed signal delays, shown in Fig. 3 (right). The second one is the distance between the real position $\left(x_{\mathrm{R}}, y_{\mathrm{R}}\right)$ of the RSU device and the estimated position $\left(x_{\mathrm{E}}, y_{\mathrm{E}}\right)$ determined by the applied method. For a single test, the precision, $P$, is expressed as follows:

$$
P=\frac{r_{\mathrm{SC}}-\sqrt{\left(x_{\mathrm{R}}-x_{\mathrm{E}}\right)^{2}+\left(y_{\mathrm{R}}-y_{\mathrm{E}}\right)^{2}}}{r_{\mathrm{SC}}}
$$

The obtained precision of $90 \%$ is based on multiple tests and is the value for the worst-case scenario.

A good practice is to try to reduce the amplitude of noise directly at the outputs of the on-board sensors of the vehicle responsible for calculation of its trajectory, for example, of the yaw rate sensor. This substantially improves the results and simplifies the computations in Stage 3 of the proposed procedure. We have already dealt with this problem during the implementation of a company automotive project, in which it was necessary to precisely filter the yaw rate signal to improve the behavior of realized active safety functions [16].

\section{CONCLUSIONS}

In this work we presented solutions which can be of importance for the implementation of novel automotive ADAS functions and their common usage in smart cities and in the ITS in the nearest future. High quality standards for such systems is expected, as the goal is to ensure full vehicle autonomy relatively soon. The obtained results are therefore important as the safety of the Intelligent Transportation System users is concerned. It is frequently stressed that this system will need support from the urban and the road infrastructures. One of the main challenges involved with this idea is the precise determination of the vehicle position with respect to the urban infrastructure, other road users and other objects.

A prototype of the proposed method was implemented in the Octave/Matlab environment and tested for different values of particular parameters (trajectories, noise, measurement frequencies, etc.). The model was investigated for different values 
of the noise and time delays - the sources of the systematic and random errors - and it was shown that the estimation of the real position of the RSU can be improved by more than $90 \%$. For test purposes, we selected relatively large values of particular distorting factors, which are rather not expected in the real environment - the radius of the area of uncertainty greater than $1.5 \mathrm{~m}$, accompanied by the noise with the amplitude at the level of $50 \%$ of the selected radius. After the calculations, the obtained deviations from real position do not exceed $2-10 \mathrm{~cm}$., which can be regarded as a satisfactory result.

Larger values of distorting factors were chosen for one more reason. Real road tests are needed to verify the reliability of the proposed method. Presently, it is not possible to carry out comprehensive tests of this type due to the lack of functioning V2I networks. That is why larger values of distorting factors were assumed to take the worst case scenario into consideration.

However, all calculations were performed assuming equal heights of the RSU and the vehicle. The problem of unequal heights would require additional calculations in three dimensions. Another solution would be equipping the devices with the information regarding their heights, which would result in less complex computations.

\section{ACKNOWLEDGEMENT}

The presented work is a part of the project: "Development of innovative technologies in the field of active safety, which will be used in advanced driver assistance systems (ADAS) and autonomous driving systems intended for mass production", under the framework of "Quick path" program, No. POIR.01.01.01-00-1398/15 (Poland).

\section{REFERENCES}

[1] O. Hassan, I. Adly, K.A. Shehata, "Vehicle Localization System based on IR-UWB for V2I Applications", 8th International Conference on Computer Engineering \& Systems (ICCES), Nov. 2013.

[10] Lianlin Zhao, Eric T. Psota, Lance C. Pérez, "A Comparison Between UWB and TDOA Systems for Smart Space Localization", IEEE International Conference on Electro/Information Technology (EIT), 2014, pp.179-193.
[2] Euro New Car Assessment Program (NCAP) - Safety Assist: https://www.euroncap.com/en/vehicle-safety/the-ratings-explained/ safety-assist/

[3] Lin Mingyao, "Transmission system for wireless electronic traffic sign and position", Patent CN1841411 (A) - 2006-10-04.

[4] Hu Jianguo, "Traffic sign wireless broadcasting system", Patent CN203070537 (U) - 2013-07-17.

[5] Kuo Hsiao-Chen, "Traffic sign wireless broadcast device", Patent TW201104632 (A) - 2011-02-01

[6] Marzieh Dashti, Mir Ghoraishi, et al., "High-Precision Time-of-Arrival Estimation for UWB Localizers in Indoor Multipath Channels", Novel Applications of the UWB Technologies, edited by Boris Lembrikov, ISBN 978-953-307-324-8, Aug., 2011.

[7] Ł. Zwirełło, Realization Limits of Impulse-Radio UWB Indoor Localization Systems, Dissertation, Karlsruher Institut für Technologie (KIT), Fakultät für Elektrotechnik und Informationstechnik, 2013.

[8] P. Merriaux, Y. Dupuis, R. Boutteau, P. Vasseur, X. Savatier, "A study of Vicon system positioning performance", Sensors, 17(7), 1591, doi: 10.3390/s17071591, 2017

[9] R. Długosz, J. Pauk, P.A. Farine "New Trends in Motion Capture Systems for Human Gait Analysis", Machine Graphics and Vision, vol. 20, no. 3, 2011, pp. 319-331.

[11] P. Karbownik, G. Krukar, A. Shaporova, et al., "Evaluation of Indoor Real Time Localization Systems on the UWB Based System Case", 2015 International Conference on Indoor Positioning and Indoor Navigation (IPIN), Banff, Alberta, Canada, Oct. 2015

[12] S. Kabil, B. Ait Essaid, A. Ait Ouahman, et al., "Analysis of UWBOFDM system for Vehicle to Infrastructure communication", $4^{\text {th }}$ International Conference on Logistics (LOGISTIQUA), June 2011, pp. 319-331.

[13] D. Dardari, N. Decarli, et al., "High-Accuracy Tracking Using Ultrawideband Signals for Enhanced Safety of Cyclists" Mobile Information Systems, Vol. 2017, Article ID 8149348, 2017.

[14] S. Kabil, R. Elassali, F. Elbahhar, et al., "Orthogonal Frequency-Division Multiplexing Ultra Wide Band System in Real Environment for Vehicle to Infrastructure Application", Journal of Computer Science, Vol. 9, Iss. 10, DOI : 10.3844/jcssp.2013.1305.1317, pp.1305-1317.

[15] J. Wang, Y. Gao, Z. Li, X. Meng, C. M. Hancock, "A Tightly-Coupled GPS/INS/UWB Cooperative Positioning Sensors System Supported by V2I Communication", Sensors, 16(7), 944; doi:10.3390/s16070944, 2016.

[16] R. Dlugosz, M. Szulc, M. Kolasa, et al., "Design and Optimization of Hardware-Efficient Filters for Active Safety Algorithms," SAE International Journal Passengers Cars - Electronic and Electrical Systems, 8(1):2015, doi:10.4271/2015-01-0152, 2015. 